\title{
Estudio de las lesiones en el judo.
}

\author{
DOI: http//dx.doi.org/10.37315/SOTOCAV20202835579 \\ ORENGA MONTOLIU S, HURTADO OLIVER V, PICAZO GABALDÓN BR, GRACIA OCHOA M, ESCRIBANO ZACARÉS S, CAPÓ SOLIVERES \\ I, VILLANUEVA DOLCET C
}

SERVICIO DE CIRUGÍA ORTOPÉDICA Y TRAUMATOLOGÍA. HOSPITAL SAN FRANCESC DE BORJA DE GANDIA. ESPAÑA.

\begin{abstract}
Resumen.
El Judo es un arte marcial cuyos orígenes proceden del ju-jutsu, creado en 1882 por el maestro Jigoro Kano, quien propuso un método que se apoyaba en técnicas que podían aplicarse en un combate real, así como practicarse con el compañero sin riesgo de lesiones. El objetivo del presente estudio es valorar la prevalencia, tipo y distribución de las lesiones sufridas. Comprobar si el sexo o el rendimiento deportivo influyen en la aparición de lesiones, determinar qué técnica interviene más frecuentemente, valorar si se producen mayoritariamente en el entrenamiento o en competición, así como analizar su forma de actuación. Se trata de un estudio descriptivo observacional transversal, en el que se solicitó participación a cuatro clubs, en el que debían responder una encuesta anónima. En cuanto a los resultados, se registraron un total de 652 lesiones, en 63 hombres y 38 mujeres. Las regiones corporales más comúnmente lesionadas fueron las extremidades superiores $254(38,95 \% \%)$. Las contusiones y los esguinces eran las lesiones más frecuentes y la técnica asociada con mayor frecuencia fue Seoi Nage. El número de lesiones fue mayor durante la competición si tenemos en cuenta el tiempo. La mayoría de las lesiones $(74,1 \%)$ fueron de características leves, con un reposo deportivo menor a una semana y únicamente un 38,95\% obligó al judoka a interrumpir la práctica deportiva. Tras el estudio realizado podemos concluir que los resultados obtenidos son comparables a la bibliografía, confirmándose los objetivos planteados.
\end{abstract}

Palabras clave: Artes marciales, Judo, Lesiones en Judo, Lesiones en las artes marciales.

\section{Summary.}

Judo is a martial art whose origins come from ju-jutsu, created in 1882 by the master Jigoro Kano, who proposed a method that was based on techniques that could be applied in real combat, as well as practiced with the partner without risk of injury. The objective of the present study is to assess the prevalence, type and distribution of the injuries suffered. Check whether sex or sports performance influences the appearance of injuries, determine which technique intervenes more frequently, assess whether they occur mostly in training or competition, and analyze how they act. This is a descriptive cross-sectional observational study, in which four clubs were asked to participate, in which they had to answer an anonymous survey. Regarding the results, a total of 652 injuries were registered, in 63 men and 38 women. The most commonly injured body regions were the upper limbs $254(38.95 \% \%)$. Contusions and sprains were the most frequent injuries and the most frequently associated technique was Seoi Nage. The number of injuries was higher during the competition if we take into account time. Most of the injuries $(74.1 \%)$ were of slight characteristics, with a sports rest of less than a week and only $38.95 \%$ forced the judoka to interrupt the sport. After the study carried out, we can conclude that the results obtained are comparable to the bibliography, confirming the stated objectives.

\author{
Correspondencia: \\ Sonia Orenga Montoliu \\ sonia.orenga@gmail.com
}




\section{Introducción}

La palabra judo proviene de dos términos japoneses: "JU" ( 柔), que significa flexibilidad y "DO” (道), camino. El término japonés puede traducirse como "camino hacia la flexibilidad". Fue creado por el maestro japonés Jigoro Kano en 1882, quien recopiló la esencia técnica y táctica de dos de las antiguas escuelas clásicas del ju-jutsu, adaptándolas. Seleccionó las técnicas que podían aplicarse en un combate real, por ser intuitivas y sencillas, así como practicarse con el compañero sin riesgo de lesiones. Se prohibieron técnicas peligrosas, como las torsiones o luxaciones de cuello, tobillo y muñecas. Dichas técnicas, así como los golpes y patadas, sólo se practican en forma de kata, reflejando la voluntad de Kano de mantener esencialmente seguro este deporte ${ }^{1}$.

\section{Material y método}

Se trata de un estudio descriptivo observacional transversal sobre las lesiones en el Judo. Se recogieron datos de los mayores de 15 años, hombres y mujeres, que practican de forma regular y que tienen más de un año de experiencia en el deporte estudiado, independientemente de su gradación.

La cumplimentación de los datos del cuestionario entregado se realizó por los propios deportistas, asesorados por el investigador principal en persona para preservar la homogeneidad de los datos. Se escogieron participantes competidores y no competidores para obtener una muestra más heterogénea. Los datos fueron recogidos durante el mes de Enero y Febrero de 2018.

El resultado de las encuestas fue trasladado a una hoja de Microsoft Excel y analizado con el programa SPSS $₫$ versión 21.

Se solicitó a los participantes en el estudio, que anotasen las lesiones sufridas a lo largo de su vida deportiva practicando judo. Se les pidió que mencionasen tantas lesiones como recordasen y que indicasen aquellas que habían ocurrido durante el transcurso de una competición, así mismo, se les solicitó que anotasen la técnica que estaban realizando (tanto de tori como de uke) en el momento de la lesión.

De un total de 108 diagnósticos distintos, se sintetizó en 16 más genéricos para un adecuado estudio estadístico, tomando las patologías más habituales observadas (Tabla I).

\section{Resultados}

Participaron en el estudio 101 deportistas, con un edad media de 26,25 años. Se observa que prevalece la participación del varón frente a la mujer con un total de 63 hombres $(63,63 \%)$ y 38 mujeres $(38,38 \%)$.

\begin{tabular}{|l|}
\hline \multicolumn{1}{|c|}{ Diagnóstico de lesiones } \\
\hline Contusión/Hematoma \\
\hline Rozaduras/Quemaduras/Heridas \\
\hline Contractura muscular \\
\hline Tendinopatías \\
\hline Esguince \\
\hline Luxación/Subluxación \\
\hline Fractura \\
\hline Rotura meniscal(Meniscopatía \\
\hline Inestabilidad/Lesión labrum/SLAP \\
\hline Lesión osteocondral/Condropatía \\
\hline Rotura muscular (fibrilar)/Microrotura \\
\hline Rotura tendinosa \\
\hline Rotura ligamentosa \\
\hline $\begin{array}{l}\text { Artropatía inflamatoria (Cambios degenerativos /Capsulitis } \\
\text { /Lesión ligamentos Interfalángica dedos /Inflamación } \\
\text { articulación) }\end{array}$ \\
\hline Latigazo cervical/rectificación cervical/esguince \\
\hline Desprendimiento de retina \\
\hline
\end{tabular}

Tabla I: Diagnóstico lesional

Del total de deportistas encuestados, 50 (49,50\%) son deportistas de alto rendimiento, mientras que 51 (50,49\%) únicamente practicaba judo de forma recreacional, obteniendo un resultado homogéneo de la muestra. De todos ellos, sólo $11(10,89 \%)$ habían participado en competiciones de katas. Se encontró el mayor número de practicantes con cinturones superiores (marrón y todos los danes de negro) con $63(62,37 \%)$ sujetos, mientras que cinturones Kyu se obtuvieron $38(37,62 \%)$ participantes.

Dentro de los datos de control de los participantes en el estudio, observamos que la técnica que había ocasionado más lesiones fue Seoi Nage con $25(24,75 \%)$ casos; que a su vez se corresponde con la técnica más utilizada por nuestros deportistas. Así mismo, la siguiente técnica que había ocasionado más lesiones fue Juji Gatame (técnica de luxación de codo), presentando hasta en 20 ocasiones $(19,80 \%)$ una subluxación del codo con lesión del complejo ligamentoso medial. Seguida de Tai Otoshi con 12 $(11,88 \%)$ lesiones.

De los 101 judokas que participaron en el estudio, informaron un total de 652 lesiones, con un promedio de 6'5 lesiones por participante a lo largo de su vida deportiva. Se calculó una tasa global de lesiones de 398 (61,04\%) entre hombres, con una tasa de lesiones de mujeres de 254 $(38,95 \%)$. No hubo diferencias estadísticamente significativas entre estas tasas de lesiones, obteniéndose un resultado semejante entre ambos sexos, con una $p=$ 0,841 .

Alrededor del $94,8 \%$ de las lesiones totales ocurrieron durante el entrenamiento, el otro $5,21 \%$ de las lesiones ocurrieron en el transcurso de una competición. 
En todos los niveles de rendimiento, las regiones corporales más comúnmente lesionadas fueron las extremidades superiores $254(38,95 \% \%)$ e inferiores 202
(30,98\%\%), específicamente lesiones en los dedos de la mano $98(15,03 \%)$ y la rodilla $55(8,43 \%)$ (Tabla II).

\begin{tabular}{|c|c|c|}
\hline Zona lesionada & $\mathbf{N}^{\circ}$ de lesiones & Porcentaje de lesiones \\
\hline Cabeza y Cuello & 141 & $21,62 \%$ \\
\hline Cabeza & 24 & $3,68 \%$ \\
\hline Cara & 49 & $7,51 \%$ \\
\hline Orejas & 31 & $4,75 \%$ \\
\hline Cuello & 37 & $5,67 \%$ \\
\hline Miembro superior & 254 & $38,95 \%$ \\
\hline Hombro & 43 & $6,59 \%$ \\
\hline Clavícula & 26 & $3,98 \%$ \\
\hline Codo & 44 & $6,74 \%$ \\
\hline Antebrazo & 11 & $1,68 \%$ \\
\hline Muñeca - Mano & 32 & $4,9 \%$ \\
\hline Pulgar & 37 & $5,67 \%$ \\
\hline Dedos trifalángicos & 61 & $9,35 \%$ \\
\hline Miembros inferiores & 202 & $30,98 \%$ \\
\hline Cadera & 11 & $1,68 \%$ \\
\hline Muslo & 21 & $3,22 \%$ \\
\hline Rodilla & 55 & $8,43 \%$ \\
\hline Tibia - Peroné & 15 & $2,3 \%$ \\
\hline Aquiles & 3 & $0,46 \%$ \\
\hline Tobillo - Pie & 50 & $7,66 \%$ \\
\hline Dedos & 47 & $7,2 \%$ \\
\hline Tronco & 55 & $8,43 \%$ \\
\hline Esternón & 7 & $1,07 \%$ \\
\hline Costillas & 19 & $2,91 \%$ \\
\hline Abdomen & 3 & $0,46 \%$ \\
\hline $\begin{array}{c}\text { Raquis dorsal y } \\
\text { lumbar }\end{array}$ & 20 & $3,06 \%$ \\
\hline Pelvis - Sacro & 6 & $0,92 \%$ \\
\hline
\end{tabular}

Tabla II: Distribución de las lesiones en el área corporal. 
De las veintitrés zonas corporales consideradas, destacan en cuanto a la frecuencia con que se dan en ellas las lesiones, en los dedos de la mano, la rodilla y el tobillo, que acaparan el $31,11 \%$ de las lesiones. El tendón de Aquiles y el abdomen son las zonas con menos lesiones, menos del $1 \%$ cada una.

La clasificación del diagnóstico general de las lesiones (Tabla III) la hemos hecho ordenándolas de mayor a menor frecuencia de presentación, y se ha encontrado que las contusiones y los esguinces son las dos lesiones más frecuentes en la práctica del judo, ya que entre ambas suponen más del $49,1 \%$ de los 16 diagnósticos considerados. Las siguientes: artropatías inflamatorias, luxaciones y rozaduras/quemaduras superan el $28,67 \%$ del total.

\begin{tabular}{|c|c|c|}
\hline $\begin{array}{c}\text { Tipo de lesiones } \\
\text { (diagnóstico general) }\end{array}$ & $\mathrm{N}^{\circ}$ de lesiones & Porcentaje de lesiones \\
\hline Contusión/Hematoma & 155 & $23,77 \%$ \\
\hline Esguince & 113 & $15,33 \%$ \\
\hline $\begin{array}{c}\text { Artropatía inflamatoria } \\
\text { (Cambios degenerativos } \\
\text { /Capsulitis /Lesión ligamentos } \\
\text { Interfalángica dedos /Inflamación } \\
\text { articulación) }\end{array}$ & 78 & $11,96 \%$ \\
\hline Luxación/Subluxación & 61 & $9,35 \%$ \\
\hline Rozaduras/Quemaduras/Heridas & 48 & $7,36 \%$ \\
\hline Fractura & 44 & $6,74 \%$ \\
\hline Contractura muscular & 38 & $5,82 \%$ \\
\hline Tendinopatías & 34 & $5,21 \%$ \\
\hline $\begin{array}{c}\text { Rotura muscular } \\
\text { (fibrilar)/Microrotura }\end{array}$ & 29 & $4,44 \%$ \\
\hline Rotura tendinosa & 14 & $2,14 \%$ \\
\hline $\begin{array}{c}\text { Latigazo cervical/rectificación } \\
\text { cervical/esguince }\end{array}$ & 14 & $2,14 \%$ \\
\hline Rotura ligamentosa & 11 & $1,68 \%$ \\
\hline Rotura meniscal/Meniscopatía & 5 & $4,95 \%$ \\
\hline $\begin{array}{c}\text { Inestabilidad/Lesión } \\
\text { labrum/SLAP }\end{array}$ & 5 & $4,95 \%$ \\
\hline $\begin{array}{c}\text { Lesión } \\
\text { osteocondral/Condropatía }\end{array}$ & 1 & $0,15 \%$ \\
\hline Desprendimiento de retina & 1 & $0,15 \%$ \\
\hline
\end{tabular}

Tabla III: Diagnóstico general

Pese a estos resultados, si analizamos de forma individual las lesiones específicas obtenemos que la lesión más frecuente en el judo es el esguince de tobillo (lesión del complejo ligamentoso lateral), con un total de 42 lesiones $(6,44 \%)$, seguido de las lesiones de los ligamentos colaterales y cambios degenerativos de los dedos de la mano a nivel de la interfalángica proximal y distal con 40 $(6,13 \%)$ casos Tabla IV.
En cuanto a la gravedad de las lesiones, la Tabla $V$ clasifica la duración de las mismas en este aspecto, según la clasificación de Fuller ${ }^{2}$. El orden según la severidad de la lesión es: muy leve (0-1 días), mínima (2-3 días), leve (4-7 días), moderada (8-28 días), severa (> 28 días), "Baja deportiva", "Lesiones catastróficas no fatales". 


\begin{tabular}{|c|c|c|c|c|}
\hline \multicolumn{5}{|c|}{ Lesiones más frecuentes } \\
\hline & $\begin{array}{l}\text { Orenga } \\
(2018)\end{array}$ & $\begin{array}{l}\text { Akoto }^{13} \\
(2017)\end{array}$ & $\begin{array}{c}\text { García }^{29} \\
(2008)\end{array}$ & $\begin{array}{l}\text { Souza }^{9} \\
(2006)\end{array}$ \\
\hline Oreja de coliflor & $4,6 \%$ & & $22 \%$ & \\
\hline $\begin{array}{l}\text { Lesión inespecífica del } \\
\text { raquis cervical }\end{array}$ & $5,98 \%$ & $4,07 \%$ & $77,7 \%$ & \\
\hline $\begin{array}{l}\text { Luxación Acromio- } \\
\text { Clavicular }\end{array}$ & $3,68 \%$ & $11,3 \%$ & $22,2 \%$ & \\
\hline $\begin{array}{c}\text { Lesiones inespecíficas } \\
\text { del hombro }\end{array}$ & $6,59 \%$ & $11,11 \%$ & & $16,37 \%$ \\
\hline Luxación glenohumeral & $1,6 \%$ & $6,2 \%$ & $8,3 \%$ & $5,45 \%$ \\
\hline Fractura clavícula & $0,46 \%$ & $5,49 \%$ & & \\
\hline $\begin{array}{l}\text { Subluxación codo } \\
\text { (lesión complejo } \\
\text { ligamentoso cubital) }\end{array}$ & $2,91 \%$ & $4,35 \%$ & & \\
\hline $\begin{array}{c}\text { Artropatía inflamatoria } \\
\text { dedos }\end{array}$ & $9,35 \%$ & & & $8,18 \%$ \\
\hline Lesión LCM de la rodilla & $3,06 \%$ & & $66,6 \%$ & $4,12 \%$ \\
\hline Lesión meniscal & $0,92 \%$ & $4,33 \%$ & $55,4 \%$ & $6,36 \%$ \\
\hline Lesión LCA de la rodilla & $1,84 \%$ & $10,73 \%$ & & $2,24 \%$ \\
\hline $\begin{array}{c}\text { Lesión complejo } \\
\text { ligamentoso lateral del } \\
\text { tobillo }\end{array}$ & $6,44 \%$ & $4,35 \%$ & $83,3 \%$ & $1,82 \%$ \\
\hline Fractura costal & $1,68 \%$ & $5,4 \%$ & & $0,91 \%$ \\
\hline
\end{tabular}

Tabla IV: Porcentaje de lesiones más frecuentes. 


\begin{tabular}{|c|c|c|}
\hline \multicolumn{3}{|c|}{ Severidad de la lesión } \\
\hline & Media & Porcentaje \\
\hline Muy leve & 388 & $59,5 \%$ \\
\hline Mínima & 10 & $1,6 \%$ \\
\hline Leve & 84 & $13 \%$ \\
\hline Moderada & 99 & $15,4 \%$ \\
\hline Severa & 68 & $10,6 \%$ \\
\hline Baja deportiva & 3 & $0,46 \%$ \\
\hline Lesiones catastróficas & & \\
\hline no fatales & 0 & $0 \%$ \\
\hline
\end{tabular}

Tabla V. Severidad de la lesión.

De un total de 652 lesiones, únicamente un 38,95\% obligó al judoka a interrumpir la práctica deportiva. Un total de 342 lesiones $(52,45 \%)$ no fueron valoradas ni tratadas por personal sanitario. Mientras que 157 (24,07\%) fueron diagnosticadas y tratadas por un Fisioterapeuta. Por otro lado, $125(19,17 \%)$ requirieron valoración e indicación médica, independientemente del tratamiento (fisioterapia o no) y únicamente $28 \quad(4,29 \%)$ judokas precisaron tratamiento quirúrgico de la lesión.

\section{Discusión}

Actualmente, con el aumento de la práctica deportiva a nivel poblacional, y el incremento en el número de competiciones, podemos afirmar que hay muy pocos deportistas que no haya sufrido ninguna lesión en sus carreras. Podemos afirmar que las lesiones en el deporte son un fenómeno complejo y multifactorial.

\section{Distribución de las lesiones en función del sexo:}

En nuestro estudio, se calculó una tasa global de lesiones de $398(61,04 \%)$ entre hombres, con una tasa de lesiones de mujeres de $254(38,95 \%)$. Si comparamos nuestros resultados, podemos afirmar que el número de lesiones es equiparable entre ambos sexos. A pesar de la diferencia en cuanto a la muestra, no se encuentra una diferencia significativa entre sexos en relación con la aparición de lesiones, por lo que no es determinante ${ }^{3-5}$

Distribución de la muestra en función del momento de la lesión:

De acuerdo con la literatura, podemos afirmar que el mecanismo más común de la lesión es durante las proyecciones $(37 \%)$ y durante la lucha de agarres $(29,7 \%)$. Probablemente esto dependa del hecho de que el judoka pasa más tiempo realizando un combate de pie $(56 \%$ del tiempo del combate) que en el trabajo de suelo $(18 \%$ del tiempo) $)^{6}$.
Además, si comparamos las lesiones en función de si ocurrieron en el transcurso del entrenamiento respecto a la competición, obtenemos: en nuestro estudio del 94,8 \% durante el entrenamiento y el $5,21 \%$ durante la competición. En el estudio de Barsottini ${ }^{7}$ del total de casos, se observó que el $71 \%$ de los procesos ocurrieron durante el entrenamiento, y solo el $20 \%$ ocurrió durante las competiciones. Estas diferencias fueron estadísticamente significativas a un nivel de significancia del $5 \%$.

No obstante, si seguimos revisando la literatura publicada, el estudio de Witkowski ${ }^{8}$, obtiene los siguientes datos: lesiones durante las competiciones (53\%), mientras que durante el entrenamiento (47\%). La mayoría de las lesiones se registraron durante los ejercicios y peleas de tachi-waza (73\%) y en ne-waza (27\%). La mitad de los encuestados que sufrieron una lesión durante el entrenamiento indicaron que ocurrió durante un randori (entrenamiento de combate). El 21\% recibió una lesión cuando practicaba deportes de equipo, el $14 \%$ cuando realizaba elementos técnicos y el $7 \%$ cuando corría y hacía ejercicio en el gimnasio. Una gran mayoría de los encuestados (94\%) dijo que la lesión había ocurrido cuando estaban en contacto directo con un oponente o compañero, mientras que sólo el $6 \%$ dijo que había sucedido mientras realizaban ejercicios individuales. En su artículo, Souza ${ }^{9}$ obtiene $43,62 \%$ de lesiones durante el entrenamiento y $49,08 \%$ de las lesiones durante el transcurso de la competición. Birrer ${ }^{10,11}$, afirma que las lesiones tienden a ser más severas en general en situaciones de torneo que durante el entrenamiento.

Por otro lado Rodríguez ${ }^{5}$ afirma en su tesis, que si tenemos en cuenta el tiempo dedicado en cada actividad, el riesgo de padecer una lesión es entre 50 y 90 veces más grande durante los combates en competiciones. Lo que supone, que en media, se han producido 60 lesiones en cada hora de competición frente a solo una lesión en cada hora de entrenamiento. Autores como Nishime ${ }^{12}$ confirman que se dan más lesiones en campeonatos, lo que coincide con lo anteriormente expuesto.

Prevalencia de las lesiones en función del rendimiento deportivo:

En nuestro estudio, no apreciamos una diferencia significativa respecto a las lesiones que presentan los judokas de alto rendimiento $377(57,82 \%)$, respecto a los amateurs $275(42,18 \%)$, con una $p<0,868$. No obstante, según el estudio de Akoto $^{13}$, aquellos atletas de alto rendimiento que participaban en competiciones, presentaron un mayor número de lesiones y gravedad de las mismas, respecto a aquellos que practicaron judo recreativamente. El riesgo de lesión depende más de la intensidad de la participación del judo que en el nivel deportivo.

\section{Análisis de los datos en función de las lesiones}

Según Birrer ${ }^{11}$, un el alto porcentaje $(63 \%)$ de las lesiones relacionadas con el arte marcial no se informan. En muchas ocasiones, por el propio desconocimiento del deportista, el umbral del dolor que presentan o que consideran 
determinadas lesiones algo "normal" y asociado a la práctica diaria del judo, hace que en gran cantidad de ocasiones, las lesiones pasen totalmente desapercibidas.

Como se puede apreciar en la Tabla $V$, las lesiones más frecuentes las podemos encontrar en el miembro superior, con alguna excepción. Si analizamos individualmente el área corporal lesionada, podremos apreciar como las lesiones sufridas por los atletas afectaron principalmente a sus extremidades superiores $(38,95 \%)$ e inferiores $(30,98 \%)$, especialmente hombro, dedos y rodilla. Se pueden observar resultados similares en los trabajos de los autores mencionados anteriormente. En un estudio prospectivo de atletas de élite, Kim et al. ${ }^{14}$ encontraron un patrón similar, con un $32 \%$ de lesiones en miembros superiores y un $39 \%$ en la Miembros inferiores. Pocecco et al. ${ }^{15}$ también especificaron las regiones más afectadas del cuerpo, especialmente la rodilla ( $28 \%$ de los casos), el hombro $(22 \%)$ y el mano y dedo $(30 \%)$.

\section{Cabeza y cuello:}

Kim et al. ${ }^{14}$ informaron que el $17 \%$ de las lesiones notificadas correspondían al raquis cervical. Las lesiones de columna y cuello pueden ocurrir, pese a que sean muy poco frecuentes, la mayoría son causadas, al recibir un golpe en la cabeza o ser proyectados y caer sobre esta misma.

El daño cervical se debe principalmente a lesiones por latigazo o compresión. Kamitani ${ }^{16}$ describió las lesiones en cabeza y cuello y en su artículo detalla que el osoto-gari fue la causa principal $(41 \%)$ de lesiones en la cabeza.

Entre los casos de lesiones en el cuello, el $60 \%$ ocurrió cuando tori intentó proyectar a un oponente, y el $40 \%$ ocurrió de uke (al ser proyectado). Una lesión típica en el cuello, es la hiperflexión de la columna cervical, que ocurre cuando un deportista intenta proyectar a su oponente mediante un uchi-mata, pero falla durante la realización de la técnica y luego cae sobre el tatami golpeándose la cabeza $^{17,18}$.

Así mismo, Akoto ${ }^{13}$ en su estudió, demostró que el prolapso del disco vertebral era una de las lesiones con una baja deportiva prolongada (después del LCA).

\section{Miembro superior:}

Las lesiones en el hombro y clavícula pueden estar relacionadas con el hecho de que, en algunas situaciones, los atletas caen sobre sus oponentes, causando sobrecarga ya que el impacto involucra su peso, fuerza y velocidad $^{9}$. De esta forma, se pueden observar distintos tipos de lesiones ocasionadas por contusiones directas sobre el tatami, ocasionalmente asociadas a una mala técnica de caída (ukemi), así como la movimientos explosivos de desequilibrio y lucha de agarres. En nuestro estudio, el 8,12 \% de las lesiones reportadas, corresponde específicamente al hombro, pudiendo diferenciar: contusiones, roturas fibrilares del deltoides, luxaciones glenohumerales, lesiones del labrum y tendinopatías del manguito de los rotadores y $5,67 \%$ en la clavícula, incluyendo contusiones, luxaciones acromio-claviculares, lucaxiones esterno-claviculares y fractura de clavícula. Pierantozzi ${ }^{17}$ describe hasta un total de $11,1 \%$ de lesiones en el hombro, resultados similares a los expuestos por Green $^{3}$, quien describe $12,53 \%$ de lesiones, y Akoto ${ }^{13}$ $17,04 \%$ en las que incluye afectación tendinosa, contusiones, cambios degenerativos y luxaciones glenohumerales.

La lesión más común en el codo, está relacionada con las técnicas de luxación, técnica de bloqueo del brazo en la que se aplica una hiperextensión con fuerza de tensión en valgo, es la lesión del ligamento colateral anterior capsular y cubital (UCL, especialmente del haz anterior). La lesión del nervio cubital y la lesión de la articulación radiocapitelar ocurren con mucha menos frecuencia pero deben considerarse $^{12}$. En nuestro estudio correspondía al 9,35\% del total de lesiones, asociado en su mayoría a la técnica juji-gatame.

Los dedos y la muñeca se someten continuamente a agresiones durante el transcurso del combate, debido a la lucha de agarres. Estos datos son corroborados por Strasser et al. ${ }^{19}$, que señala dos características: la primera se relaciona con la manifestación aguda: contusiones, esguinces, distensiones provocados por caídas y lesiones provocadas en el momento en que la mano del oponente es sacada del judogi; y el segundo, crónico, debido al entrenamiento prolongado y exhaustivo de kumi-kata lleva a cambios degenerativos en las articulaciones de los dedos $^{9}$.

En toda la bibliografía revisada, no informan de ninguna lesión relacionada con las técnicas de Kote Waza (técnicas de control y luxación de la muñeca), aplicadas durante la práctica del kata.

\section{Rodilla:}

Varios estudios han indicado que la rodilla es la región más frecuentemente lesionada durante la práctica del judo. ${ }^{4,14,20}$ Las lesiones de rodilla más comunes en nuestra población fueron les lesiones del ligamento colateral medial $(36,36 \%$ del total de lesiones en la rodilla), seguidas de las roturas del LCA $(21,81 \%)^{21}$. Las lesiones de rodilla, ocurren con frecuencia secundariamente al alto estrés en valgo en la articulación ${ }^{1}$. Sólo el artículo publicado por Akoto $^{13}$, ha demostrado que la rotura del Ligamento cruzado anterior, es la lesión de rodilla más frecuente y grave en el judo. Sus datos sugieren la importancia de desarrollar un programa de prevención de lesión del ligamento cruzado anterior similar a los que se han introducido en otros deportes.

Según el estudio de Koshida et al. ${ }^{20}$, el mecanismo lesional más frecuente en las lesiones del LCA, fue el contacto directo con la extremidad inferior. Además, asocia las técnicas: osoto-gari, kosoto-gari y harai-goshi como una causa común en dichas lesiones, estas técnicas tienen características técnicas similares y son ejecutadas por una pierna de barrido que hace contacto directo. El estrés en valgo y luego la fuerza de rotación aplicada a la pierna del participante por la acción del oponente en la fase de lanzamiento fueron los factores causales. Estas rotaciones, 
cuando se realizan abruptamente, provocan que los movimientos excedan los límites de funcionalidad articular ${ }^{9}$.

Así mismo, el riesgo de lesión durante la realización de taiotoshi parece ser mayor cuando la articulación de la rodilla del judoka está en una posición de extensión completa en la fase preparatoria ${ }^{20}$. Kasahara et al. $^{22}$, afirman que es importante prestar mucha atención a las lesiones de la rodilla y que la fuerza muscular de la extensión de la rodilla es crucial para su prevención. Se considera a las técnicas basadas en seoi-nage como de riesgo para las rodillas del tori en todas las categorías ${ }^{17}$ El alto impacto al realizar la variante de seoi-nage de rodillas, conocida como seoi otoshi, fue demostrado por Montero ${ }^{23}$.

Pie y tobillo:

La lesión más descrita en nuestro estudio, fue el esguince de tobillo, con afectación del complejo ligamentoso lateral en un $6,44 \%$. Hay que tener en cuenta que este deporte se practica descalzo sobre tatami, que es blando e irregular con el uso. No obstante, no se ha encontrado en la literatura ningún estudio que haga referencia a esta patología en el judo.

Raquis lumbar:
Las lesiones a nivel del raquis lumbar, se relacionaban con frecuencia a rotaciones extremas y desequilibrios al realizar las proyecciones. Un estudio sobre la espondilosis lumbar mostró que los judokas eran significativamente propensos $(20 \%)$ a sufrir este tipo de lesiones. Con frecuencia ocurría debido a movimientos repetitivos del tronco ${ }^{14,24}$. Okada et al. $^{25}$ advirtieron una prevalencia de dolor lumbar inespecífico en $35 \%$ y hallazgos radiológicamente anormales en $82 \%$ de un grupo de los atletas de judo universitarios japoneses; el $56 \%$ presentaban cambios degenerativos del disco intervertebral ${ }^{26}$.

Miscelánea:

En nuestro estudio, hemos podido observar varias lesiones que, aunque infrecuentes, son de una relativa importancia, ya que implicaron el cese de la actividad deportiva para estos judokas, por recomendación médica. Por un lado, presentamos un caso de desprendimiento de retina tras una proyección y caída muy fuerte contra el suelo, descrita por Norton ${ }^{27}$ en la literatura.

Presentamos a su vez un caso de rotura de los tendones isquiosurales a nivel proximal, en la tuberosidad isquiática, detallada por Kurosawa ${ }^{28}$.

\begin{tabular}{|c|c|c|c|c|}
\hline \multicolumn{5}{|c|}{ Localización anatómica de la lesión } \\
\hline & $\begin{array}{c}\text { Cabeza y } \\
\text { cuello }\end{array}$ & $\begin{array}{c}\text { Miembro } \\
\text { superior }\end{array}$ & Miembro inferior & $\begin{array}{c}\text { Tronco y } \\
\text { pelvis }\end{array}$ \\
\hline Orenga, 2018 $^{2}$ & $21,62 \%$ & $38,62 \%$ & $30,95 \%$ & $8,43 \%$ \\
\hline Akoto $^{14}, \mathbf{2 0 1 7}$ & $4,07 \%$ & $38,65 \%$ & $42,15 \%$ & $5,4 \%$ \\
\hline Barsottini $^{\prime}, \mathbf{2 0 0 6}$ & $1 \%$ & $37 \%$ & $53 \%$ & $9 \%$ \\
\hline Bierrer $^{\mathbf{1 0}}, \mathbf{1 9 8 8}$ & $6,5 \%$ & $26,8 \%$ & $46,9 \%$ & $18,2 \%$ \\
\hline Green $^{3}, \mathbf{2 0 0 6}$ & $18,78 \%$ & $35,4 \%$ & $19,72 \%$ & $7,27 \%$ \\
\hline Kim $^{15}, \mathbf{2 0 1 5}$ & $5,6 \%$ & $29,8 \%$ & $41,2 \%$ & $23,4 \%$ \\
\hline Kujala $^{4}, \mathbf{1 9 9 5}$ & $9,9 \%$ & $37,6 \%$ & $38,6 \%$ & $9,7 \%$ \\
\hline Pierantozzi $^{\mathbf{1 6}}, \mathbf{2 0 0 9}$ & $18,5 \%$ & $44,4 \%$ & $25,9 \%$ & \\
\hline Pieter $^{6}, \mathbf{2 0 0 3}$ & $33,12 \%$ & $28,26 \%$ & $21,41 \%$ & \\
\hline Pieter $^{1 /}, \mathbf{2 0 0 5}$ & $8 \%$ & $28 \%$ & $44 \%$ & $20 \%$ \\
\hline Souza $^{9}, \mathbf{2 0 0 6}$ & $0,91 \%$ & $45,46 \%$ & $40,91 \%$ & $4,55 \%$ \\
\hline Witkowski $^{\mathbf{8}}, \mathbf{2 0 1 2}$ & $16,66 \%$ & $43,33 \%$ & $40 \%$ & \\
\hline
\end{tabular}

Tabla VI: Porcentaje de lesiones según distribución corporal.

\section{$\underline{\text { Técnica asociada con más frecuencia a las lesiones }}$}

Vemos que la técnica que ocasiona con más frecuencia lesiones corresponde al Seoi Nage en un $24,75 \%$ y el Tai otoshi en el $22 \%$ de los casos, seguida de Juji Gatame
$19,80 \%$, causando una subluxación del codo y Tai Otoshi, $11,88 \%$ lesiones. El $22 \%$ de los atletas no recordaron el golpe asociado ${ }^{7}$. 
$\underline{\text { Severidad de las lesiones y tratamiento }}$

En la tabla VII especificamos el tiempo de reposo deportivo y la clasificación de la severidad de las lesiones.

\begin{tabular}{|c|c|c|c|c|c|c|}
\hline & Muy leve & Mínima & Leve & Moderada & Severa & $\begin{array}{c}\text { Baja } \\
\text { deportiva }\end{array}$ \\
\hline Orenga, 2018 $^{*}$ & $59,5 \%$ & $1,6 \%$ & $13 \%$ & $15,4 \%$ & $10,6 \%$ & $0,46 \%$ \\
\hline Barsottini $^{\prime}, \mathbf{2 0 0 6}$ & \multicolumn{7}{|c|}{$10 \%$} & $9 \%$ & $63 \%$ & \\
\hline Birrer $^{10}, \mathbf{1 9 8 8}$ & $45 \%$ & $30 \%$ & $12 \%$ & $7,7 \%$ & $4,7 \%$ & $0,6 \%$ \\
\hline Rodríguez $^{12}, \mathbf{2 0 1 5}$ & \multicolumn{2}{|c|}{$21 \%$} & $51 \%$ & $16 \%$ & $12 \%$ & \\
\hline
\end{tabular}

Tabla VII. Severidad de las lesiones.

Según Rodríguez ${ }^{5}$ en su estudio, más de la mitad de las lesiones registradas (58\%) obligaron al Judoka a interrumpir la práctica deportiva. Analizando el tiempo de reposo deportivo, observamos que la mitad de los deportistas que precisan reposo para su lesión, lo hacen ente 1 y 2 semanas. Un $82 \%$ de las lesiones registradas precisaron tratamiento fisioterapéutico, respecto al $24,07 \%$ de nuestro estudio. Únicamente el 4,29\% del total de lesiones, precisaron tratamiento quirúrgico, obteniendo resultados similares a los presentados por Rodríguez ${ }^{5}$.

Podemos afirmar por los datos obtenidos, que la mayoría de judokas se abstienen a consultar por la mayoría de sus lesiones $(52,45 \%)$, ya que no las consideran importantes ${ }^{11}$. La información inferior de los datos de lesiones es un problema, ya que no permite una estimación precisa de los riesgos y la seguridad $y$, a veces, pone al atleta involucrado en peligro de lesiones importantes.

El judo, como todos aquellos deportes que exigen un esfuerzo extremo, expone al deportista a un alto riesgo de lesiones, presentando un alto porcentaje de las mismas. No obstante, pese a ser un deporte de contacto, no aparece ninguna lesión letal o muy grave, por lo que podemos afirmar que el judo es un deporte seguro ${ }^{13}$. Los resultados de este estudio retrospectivo respaldan los datos de investigaciones similares.

\section{Limitaciones}

Una de las limitaciones de los datos retrospectivos utilizados en este estudio es que las lesiones y sus severidades fueron autoinformadas. El análisis de los resultados debe considerar que los atletas entrevistados no pudieron recordar todas las lesiones sufridas, ya que se preguntaba por el total de lesiones a lo largo de su vida deportiva. Aunque esta limitación implica un registro menor de diagnósticos específicos, el objetivo principal de nuestro estudio fue presentar frecuencias específicas de la región y del tipo de lesiones deportivas.

Las frecuencias de las lesiones no estaban relacionadas con un período de tiempo definido. Por este motivo, solo se pueden informar las proporciones de lesiones (y no las tasas de lesiones).

La mejor forma de evaluar las exposiciones deportivas sería análisis prospectivo. Sin embargo, nuestro estudio no buscó comparar las tasas de lesiones de judo con las tasas de otros deportes; en cambio, buscó realizar un análisis transversal de lesiones en el judo. Por lo tanto, este estudio puede servir como base para futuras investigaciones prospectivas sobre exposiciones y posibles estrategias de prevención. 


\section{Conclusiones}

Tras el estudio realizado y como respuesta a los objetivos planteados podemos concluir que:

1. La lesión predominante fueron las contusiones, seguido de los esguinces, artropatías inflamatorias y luxaciones.

2. La zona más comúnmente lesionada, tanto miembros superiores como inferiores presentan resultados equiparables en la bibliografía estudiada, no obstante, en nuestro estudio predominan las lesiones en el miembro superior.

3. No se ha encontrado en la muestra estudiada una influencia del sexo o el rendimiento deportivo en la presencia de lesiones.

4. La mayoría de las lesiones ocurren durante el entrenamiento, no obstante, como se ha podido observar, sería más correcto considerar que el número de lesiones es mayor durante la competición si tenemos en cuenta el tiempo de entrenamiento respecto al de competición.

5. La técnica en la que se asocian el mayor número de lesiones es el seoi nage, con un resultado comparable a la bibliografía.

6. La consulta médica en las lesiones reportadas es muy inferior a la esperada por el tipo de lesiones que asocian, así como el reposo deportivo, únicamente un $38,95 \%$ de las lesiones obligó al judoka a interrumpir la práctica deportiva.

7. Si analizamos el tratamiento recibido por el judoka, un total de $52,45 \%$ lesiones, no fueron valoradas ni tratadas por personal sanitario. Mientras que $24,07 \%$ fueron diagnosticadas y tratadas por un fisioterapeuta. Por otro lado, un $19,17 \%$ requirieron valoración e indicación médica, independientemente del tratamiento y únicamente el $4,29 \%$ de las lesiones precisaron tratamiento quirúrgico de la lesión. 


\section{Bibliografía}

1. Villamón-Herrera M. Historia del Judo y de las artes marciales. In: Rodríguez-Rodríguez LP, editor. Compendio histórico de la actividad física y el deporte. Barcelona: Masson; 2003.

2. Fuller C, Molloy M, Bagate; Bahr R, Brooks JHM, Donson H et al. Procedures for studies of injuries in rugby unión. Medicine 2007; 41:328-32.

3. Green C, Petrou M, Fogarty-Hover MLS, Rolf CG. Injuries among judokas during competition. Scand J Med Sci Sports 2007; 17(3):20510.

4. Kujala M, Taimela S, Taimela S, Antti-Poika I, Orava S, Tuominen R, et al. Acute injuries in soccer, ice hockey, volleyball, basketball, judo, and karate: Analysis of national registry data. BMJ. 1995; 311:1465-8.

5. Rodríguez EM. Alonso, MR (dir). Epidemiología de lesiones en la práctica del judo en alto nivel. Universidad Alfonso X El Sabio, Madrid. 2015.

6. Pieter W, James G. Injury rates in adult elite Judoka. Biology of Sport 2003; 20(33):139-46.

7. Barsottini D, Guimaraes A, Morais P. Relationship between techniques and injuries among judo practitioners. Revista Brasileira de Medicina do Esporte. 2006; 12:48-51.

8. Witkowski K, Maśliński J. Causes of injuries in young female judokas. Archives of Budo. 2012; 8(2):109-16.

9. Souza M, Monteiro H, Del Vecchio F. Goncalves A. Referring to judo's sports injuries in São Paulo State Championship. Science \& Sports. 2006; 21(5): 280-4.

10. Birrer R, Halbrook S. Martial arts five year national survey. Am J Sports Med 1988; 16(4):408-10.

11. Birrer R, Birrer C. Unreported injuries in the Martial Arts. Br J Sports Med 1983; 17(2):131-3.

12. Nishime R. Martial arts sports medicine: Current issues and competition event coverage. Curr Sports Med Rep 2007; 6(3):162-69.

13. Akoto R, Lambert C. Epidemiology of injuries in judo: a cross-sectional survey of severe injuries based on time loss and reduction in sporting level. Br J Sports Med. 2017; 0:1-8.

14. Kim K, Park K, Lee J, Kang BY. Injuries in national Olympic level judo athletes: An epidemiological study. Br J Sports Med 2015; 49(17):1144-50.

15. Pocecco E, Ruedl G, Stankovic N, Sterkowicz S, Boscolo Del Vecchio F, Gutiérrez-García C. Injuries in judo: A systematic literature review including suggestions for prevention Br J Sports Med 2003; 47(18):1139-43.

16. Kamitani T, Nimura Y, Nagahiro YS, Miyazaki S, Tomatsu T. Catastrophic head and neck injuries in judo players in Japan from 2003 to 2010. Am J Sports Med 2013; 41(8):1915-21.

17. Pierantozzi E, Muroni R. Judo high level competitions injuries. Medit J Musc Surv. 2009; 17:26-9.

18. Pieter W. Martial Arts Injuries. Sports Med Sport Sci 2005. 48:59-73.

19. Strasser P, Hauser M, Häuselmann HJ, Michel BA, Frei A, Stucki G. Traumatic finger polyarthrosis in judo athletes: a follow-up study. Z Rheumatol. 1997; 56:342-50.

20. Koshida S, Deguchi T, Miyashita K, Iwai K, Urabe Y. The common mechanisms of anterior cruciate ligament injuries in judo: A retrospective analysis Br J Sports Med 2010; 44(12):856-61.

21. Majewski M, Susanne H, Klaus S. Epidemiology of Athletic knee injuries: A 10-year study. The Knee. 2006; 13:184-8.

22. Kasahara D, Martin C, C. Humberstone, Yamamoto T, Nakamura T. Classification of sports injuries in Japanese university judo players and analysis of associated physical fitness characteristics. Journal of Science and Medicine in Sport. 2015; 19(e50).

23. Montero C, López J. Impacto producido por la técnica seoi-otoshi. Relación con años de práctica y grado en judo. Rev Artes Marciales Asiát 2014; 9(1):32-41.

24. Sakai T, Sairyo K, Suzue N, Kosaka H, Yasui N. Incidence and etiology of lumbar spondylolysis: review of the literatura. J Orthop Sci 2010; 15:281-8. 
ORENGA MONTOLIU S y COLS. Estudio de las lesiones en el judo.

25. Okada T, Nakazato K, Iwai K, Tanabe M, Irie K, Nakajima H. Body Mass, Nonspecific Low Back Pain, and Anatomical Changes in the Lumbar Spine in Judo Athletes. J Orthop Sports Phys Ther 2007; 37(11):688-93.

26. Robinson Y, Kayser R, Ertel W, Heyde CE. Traumatic cervical instability in martial arts. Scand J Med Sci Sports 2007; 17(1):92-3.

27. Norton ML, Safrin M, Cutler P. Medical aspects of Judo. N Y State J Med 1967; 67(12):1750-2 [Abstract]

28. Kurosawa H, Nakasita K, Nakasita H, Sasaki S, Takeda S. Complete avulsion of the hamstring tendons fron the ischial tuberosity. A report of two cases sustained in Judo. Br J Sports Med 1996; 30:72-4.

29. García S. Miguel F, Martín P, Gutiérrez G (dir). Lesiones en artes marciales chinas frente a otros deportes de lucha individuales. Universidad Complutense de Madrid. 2014. 